\title{
Two Higgs Doublets Model in Gauge-Higgs Unification framework
}

\author{
We-Fu Chang* \\ Department of Physics, National Tsing Hua University, HsinChu 300, Taiwan and \\ Physics Division, National Center for Theoretical Sciences, HsinChu 300, Taiwan \\ Sin Kyu Kang \\ School of Liberal Arts, Seoul-Tech., Seoul 139-743, Korea and \\ Institute of Convergence Fundamental Studies, Seoul-Tech., Seoul 139-743, Korea \\ Jubin Park \\ Department of Physics, National Tsing Hua University, HsinChu 300, Taiwan
}

\begin{abstract}
We discuss the realization of two Higgs doublets model in the framework of 6 dimensional GaugeHiggs Unification model with a simple Lie group $G_{M}$. Two Higgs $S U(2)_{L}$ doublets can emerge at the low energy effective theory, and the quartic coupling terms in the scalar potential, essential for the electroweak symmetry breaking, are now $G_{M}$ gauge invariant and permissive. A realistic two Higgs doublets model can possibly be obtained only when two of the root vectors associated with the would-be Higgs doublets and the root vector for $S U(2)_{L}$ form an isosceles triangle with vertex angle either of $\pi / 3, \pi / 2$, or $2 \pi / 3$. Moreover, depending on $G_{M}$, the scalar potential of resulting two Higgs doublets model can admit only a few limited forms. The mass spectrum of the physical Higgs and the weak mixing angle are briefly discussed.
\end{abstract}

PACS numbers: 11.10.Kk, 11.15.-q, 12.10.-g

\section{INTRODUCTION}

In the Gauge-Higgs Unification(GHU) models, the vector fields of gauge group $G_{M} \supset S U(2)_{L} \times U(1)_{Y}$ propagate in $(4+\mathrm{d})$-dimensional spacetime. The gauge components in the $d$ compactified extra spatial dimensions behave like scalar fields below the compactification scale [1]. With properly chosen gauge symmetry and orbifolding boundary conditions(BC), an effective scalar $S U(2)_{L}$ doublet can emerge at the low energy and play the role of Standard Model(SM) Higgs. Hence, we have no need of introducing a fundamental scalar. Due to the higher dimensional gauge symmetry, the $d$ extra scalar fields are massless. The spontaneous electroweak symmetry breaking(EWSB) in SM can be triggered by the quantum corrections with the Wilson loop in the non-simple connected space [2]. The notorious gauge hierarchy problem associated with a fundamental Higgs boson can be thus alleviated. For instance, a Higgs doublet could arise from a 5 dimensional(5D) $S U(3)$ electroweak gauge theory on the $S_{1} / Z_{2}$ orbifold [3]. However, for $d=1$, the quartic coupling term in the scalar potential must be generated by some symmetry breaking quantum corrections for it vanishes at tree-level as well. When $d \geq 2$, the quartic coupling terms in the scalar potential, which arise from the square of field strength, are gauge invariant by construction. Moreover, it is possible to generate multi scalars at the low energy $[4]$.

In this paper, we focus on the realization of two Higgs doublets model (2HDM) in 6 dimensional(6D) GHU models, bearing in mind that (1) 2HDM predicts $\rho \equiv M_{W}^{2} /\left(M_{Z}^{2} \cos ^{2} \theta_{W}\right)=1$ after EWSB at tree-level, and (2) $d=2$ is the minimal requirement to yield two Higgs ( not limited to $S U(2)_{L}$ doublets ). We shall exhaust all possible simple Lie groups for $G_{M}$ and examine the resulting quartic coupling terms of Higgs potential, denoted as $V_{4}$, which is now completely determined by group theory at tree-level. It is a delightful surprise to us that $V_{4}$ can admit only a few forms for all possible Lie groups. Our key finding is that, to successfully generate a $2 \mathrm{HDM}$ at low energy, only the root vectors of $G_{M}$ associated with the would-be Higgs doublets and the root vector for $S U(2)_{L}$ form an isosceles triangle with vertex angle either of $\pi / 3, \pi / 2$, or $2 \pi / 3$. Moreover, $V_{4}$ solely depends on the vertex angle. Our result is summarized in Table-II. On the other hand, the quadratic terms of Higgs potential, denoted as $V_{2}$, are assumed to be generated by some symmetry breaking mechanism and they cannot be fixed by the gauge symmetry. However, by using the physical Higgs mass spectrum, one can parameterize $V_{2}$ phenomenologically and bypass the question of their origin. Finally, it is a well known difficulty to construct a GHU model with the weak mixing $\sin ^{2} \theta_{W}$ close to $1 / 4$ at tree-level $[5,6]$. In the phenomenology section, we discuss two possible remedies, by including either the brane kinetic term $(\mathrm{BKT})[$,, 8$]$ or an extra $U(1)$ factor, to address this problem and the consequent modification to the Higgs mass spectrum.

\section{GROUP THEORY ANALYSIS}

Following [5], we adopt the standard convention for Lie group that $\left[H_{i}, H_{j}\right]=0,\left[\vec{H}, E_{\alpha}\right]=\vec{\alpha} E_{\alpha},\left[E_{\alpha}, E_{-\alpha}\right]=$ 
$\vec{\alpha} \cdot \vec{H}$, and $\left[E_{\alpha}, E_{\beta}\right]=N_{\alpha, \beta} E_{\alpha+\beta}$. Here, $H$ and $E_{\alpha}$ are the Cartan and root generators of $G_{M}$ respectively. The structure constant, $N_{\alpha, \beta}$, is given by $N_{\alpha, \beta}^{2}=n(m+1)(\vec{\alpha}$. $\vec{\alpha}) / 2$ [9]. Moreover, we take the following normalization for $H$ and $E$ : $\operatorname{tr} H_{i} H_{j}=\delta_{i j}, \operatorname{tr} E_{\alpha} E_{\beta}=\delta_{\alpha+\beta, 0}$, and $\operatorname{tr} E_{\alpha} H_{i}=0$. The two extra compactified spatial coordinates, $x^{5}$ and $x^{6}$, can be conveniently described by a complex coordinate $z=\left(x^{5}+i x^{6}\right) / \sqrt{2}$ and its conjugate $\bar{z}$. Accordingly, we work with $A_{z} \equiv\left(A_{5}-i A_{6}\right) / \sqrt{2}$, the associated gauge field components in $z$, where $A_{z}=A_{z}^{a} T^{a}$ and $T^{a}$ is the group generator.

By imposing the proper orbifolding BC's, the remaining zero modes of $A_{z}$, the would-be scalars, are the gauge components associated with the unbroken group generators $E_{\beta, \gamma}$ while their $4 \mathrm{D}$ gauge components possess no zero modes. Unless further state, the notation $A_{z}$ is recycled to collectively signify these zero modes which carry one mass dimension,

$$
A_{z}=\frac{1}{2} h_{u} E_{\beta}+\frac{1}{2} h_{d} E_{\gamma}+\frac{1}{2} h_{u}^{\prime} E_{-\beta}+\frac{1}{2} h_{d}^{\prime} E_{-\gamma} .
$$

And two would-be-Higgs-doublets can be built up in the following way:

$$
H_{1}=\frac{1}{\sqrt{2}}\left(\begin{array}{c}
h_{u}^{\prime} \\
h_{d}^{\prime}
\end{array}\right), H_{2}=\frac{1}{\sqrt{2}}\left(\begin{array}{c}
-h_{d} \\
h_{u}
\end{array}\right) .
$$

The SM $S U(2)_{L}$ and $U(1)_{Y}$ groups must be embedded into $G_{M}$. If the root vector of the would-be SM $S U(2)_{L}$ is denoted as $\vec{\alpha}$, the corresponding generators read $J_{0}=$ $\frac{1}{|\vec{\alpha}|^{2}} \vec{\alpha} \cdot \vec{H}, \quad J_{+}=\frac{\sqrt{2}}{|\vec{\alpha}|} E_{\alpha}$, and $J_{-}=\left(J_{+}\right)^{\dagger}$. The would-be hypercharge generator $Y$ has to be a linear combination of Cartan generators and denoted as $\vec{y} \cdot \vec{H}$. Since the SM group is $S U(2)_{L} \times U(1)_{Y}$, hence $\vec{y} \cdot \vec{\alpha}=0$. The SM gauge bosons correspond to the zero modes of the gauge fields associated with these generators given as,

$$
A_{\mu}=W_{\mu}^{+} E_{\alpha}+W_{\mu}^{-} E_{-\alpha}+W_{\mu}^{0} \vec{\alpha} \cdot \vec{H}+B_{\mu} \vec{y} \cdot \vec{H} .
$$

In [6], the phenomenologically viable embedding of the SM electroweak groups into $G_{M}$ has been studied, where the normalization of $\vec{y}$ is fixed such that the SM Higgs doublet carries hypercharge $1 / 2\left(Q=T_{3}+Y\right)$. The root vectors $\vec{\alpha}$ and $\vec{y}$ we adopt from [6] are listed in the first two columns in Table II From the commutators of $E_{\beta, \gamma}$, one has,

$$
\vec{\gamma}+\vec{\alpha}=\vec{\beta} \text { or }(\vec{\beta}-\vec{\alpha}=\vec{\gamma}) .
$$

Since $h_{u}$ and $h_{d}$ transform into each other within one $S U(2)_{L}$ doublet, the magnitudes of the two root vectors should be the same, $|\vec{\beta}|=|\vec{\gamma}|$. Same requirement applies to the pair of $h_{u}^{\prime}$ and $h_{d}^{\prime}$. From Eq.(44), the three root vectors, $\vec{\alpha}, \vec{\beta}$, and $\vec{\gamma}$ form an isosceles triangle lying on a plane in the root space. A trivial geometrical relation follows that

$$
|\vec{\beta}| \sin \frac{\theta}{2}=\frac{|\vec{\alpha}|}{2}
$$

TABLE I: Vertex angles, isosceles triangles, and candidate simple Lie groups for the possible realizations of $2 \mathrm{HDM}$.

\begin{tabular}{|c|c|c|c|}
\hline$\theta_{\vec{\beta}, \vec{\gamma}}$ & $|\vec{\beta}|$ & candidate groups & type of triangle \\
\hline \hline $60^{\circ}$ & $|\vec{\alpha}|$ & $A_{n}, D_{n}, G_{2}, F_{4}, E_{6,7,8}$ & equilateral \\
\hline $90^{\circ}$ & $\frac{|\vec{\alpha}|}{\sqrt{2}}$ & $B_{n}, C_{n}, F_{4}$ & right isosceles \\
\hline $120^{\circ}$ & $\frac{|\vec{\alpha}|}{\sqrt{3}}$ & $G_{2}$ & obtuse isosceles \\
\hline
\end{tabular}

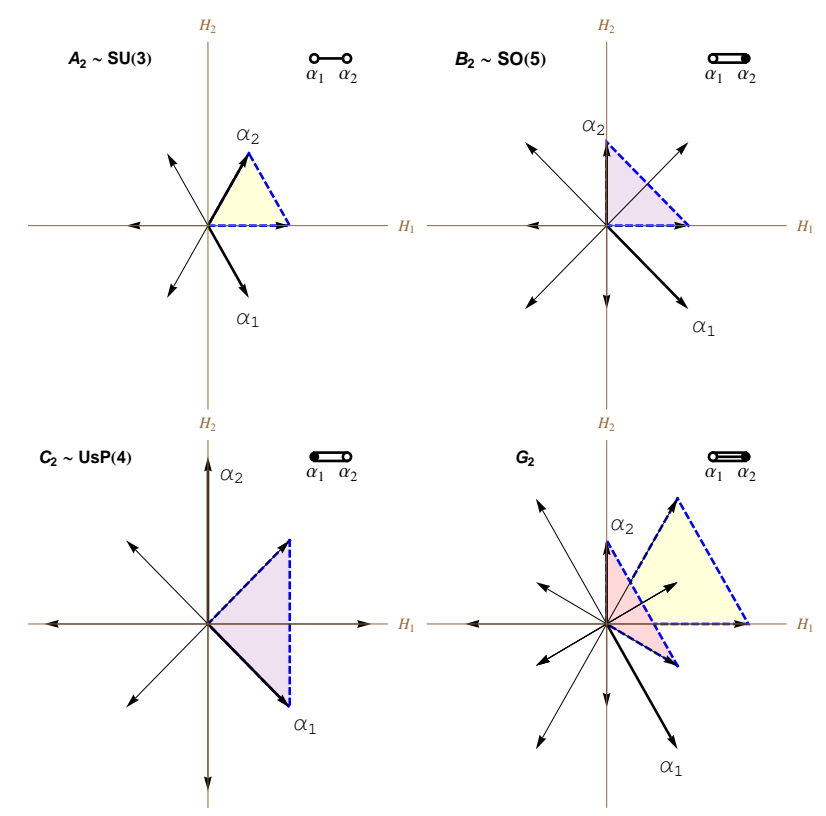

FIG. 1: Root diagrams, Dynkin diagrams and corresponding triangles of $A_{2}$ (left upper), $B_{2}$ (right upper), $C_{2}$ (left bottom) and $G_{2}$ (right bottom) where yellow, purple and red triangles represent the equilateral, right isosceles and obtuse isosceles triangles, respectively.

where $\theta$ is an angle between $\vec{\beta}$ and $\vec{\gamma}$. For a simple Lie group, $\theta$ can take only three possible values: either $\pi / 3, \pi / 2$, or $2 \pi / 3$. Hence, the original group theory problem of embedding the 2HDM in GHU model with gauge symmetry $G_{M}$ is now equivalent to looking for the existence of any equilateral, right isosceles, or obtuse isosceles triangles in the root diagram of $G_{M}$.

Once the root $\vec{\alpha}$ is given, one only needs to look up the corresponding Dynkin diagram and find out which simple root is adjacent to $\vec{\alpha}$. Next, one looks for the special isosceles triangle in the 2-dimensional space spanned by the two simple roots. In Table I] we list all possible realizations of $2 \mathrm{HDM}$, by employing Eqs.(415), in various Lie groups. Note that all groups, except $G_{2}$ and $F_{4}$, have only one possible angle between the two root vectors for the would-be Higgs doublets.

We illustrate the finding by four rank-2 groups, $A_{2}, B_{2}, C_{2}$, and $G_{2}$, which can be diagrammatically sum- 
marized in the self-explanatory Fig 1. Here, the triangles formed by the corresponding root vectors, $\vec{\alpha}, \vec{\beta}$, and $\vec{\gamma}$, are highlighted by color shades. Note that there are two distinct isosceles triangles can be drawn with $\vec{\alpha}$ in the root diagram for $G_{2}$. Therefore, there are four possible forms for $V_{4}$ (two reds, two yellows, red with yellow on one side, and red with yellow on the opposite sides) in the $G_{2}$-based GHU model. Since the root $\vec{\alpha}$ for SM $S U(2)_{L}$ is always at the end of the Dynkin diagram, the analysis for $G_{M}$ with rank higher than two is no more complicated than those displayed in Fig 1.

\section{HIGGS POTENTIAL AND WEAK MIXING}

The scalar quartic coupling terms in 2HDM arise from the 6-dimensional gauge field strength square, $g_{4 D}^{2} \operatorname{tr}\left[A_{Z}, A_{Z}^{\dagger}\right]^{2}$,

$$
\begin{aligned}
V_{4}= & \frac{g_{4 D}^{2} \vec{\beta}^{2}}{4}\left\{\left(\left|h_{d}\right|^{2}-\left|h_{u}^{\prime}\right|^{2}\right)^{2}+\left(\left|h_{u}\right|^{2}+\left|h_{d}^{\prime}\right|^{2}\right)^{2}\right. \\
& +2\left(\tilde{N}_{-\gamma, \beta}^{2}+1\right)\left(\left|h_{u}\right|^{2}\left|h_{d}\right|^{2}+\left|h_{u}^{\prime}\right|^{2}\left|h_{d}^{\prime}\right|^{2}\right) \\
& +2\left(\tilde{N}_{\gamma, \beta}^{2}-\cos \theta\right)\left(\left|h_{d}\right|^{2}\left|h_{d}^{\prime}\right|^{2}+\left|h_{u}\right|^{2}\left|h_{u}^{\prime}\right|^{2}\right) \\
& \left.+2\left(\tilde{N}_{-\gamma, \beta}^{2}+\tilde{N}_{\gamma, \beta}^{2}\right)\left(h_{d} h_{u}^{*} h_{u}^{\prime} h_{d}^{\prime *}+h_{d}^{*} h_{u} h_{u}^{\prime *} h_{d}^{\prime}\right)\right\}(6)
\end{aligned}
$$

where $\tilde{N}_{\gamma, \beta}^{2} \equiv N_{\gamma, \beta}^{2} / \vec{\beta}^{2}$. Also, Eqs. (415) and the relations $N_{\gamma, \beta}=-N_{\beta, \gamma}=-N_{-\alpha,-\gamma}$ have been used to arrive in the above expression. Unsurprisingly, the resulting Higgs potential is completely determined by the gauge group $G_{M}$ for a given root vector $\vec{\alpha}$ for SM $S U(2)_{L}$.

Eq.(6) can be written in a much more compact form as:

$$
V_{4}\left(H_{1}, H_{2}\right)=\frac{1}{2} \lambda\left(\left|H_{1}\right|^{2}-\left|H_{2}\right|^{2}\right)^{2}+\frac{1}{2} N \lambda\left|H_{1}^{\dagger} H_{2}\right|^{2},(7)
$$

As mentioned in the introduction, the Higgs quadratic couplings in GHU model must be generated radiatively by some symmetry-breaking mechanism [12]. The resulting quadratic couplings are highly model dependent. Here, we take a bottom-up approach and treat all quadratic couplings as phenomenological parameters. Then the full $S U(2)$ invariant scalar potential of $2 \mathrm{HDM}$ reads,

$$
V=m_{1}^{2}\left|H_{1}\right|^{2}+m_{2}^{2}\left|H_{2}\right|^{2}-m_{12}^{2}\left(H_{1}^{\dagger} H_{2}+H_{2}^{\dagger} H_{1}\right)+V_{4} .
$$

Based on this, a textbook EWSB analysis can be performed straightforwardly. We denote the vacuum expected values as $\left\langle H_{1}^{0}\right\rangle=v_{1}$ and $\left\langle H_{1}^{0}\right\rangle=v_{2}$ with where $\lambda=|\vec{\beta}|^{2} g_{4 D}^{2}$ and $N$ is an integer depending on the group $G_{M}$, as shown in Table III In Eq.(7), $H_{1}$ and $H_{2}$ have the desired hypercharge either $Y=1 / 2$ or $Y=-1 / 2$. Geometrically speaking, two identical or the mirror pair root triangles are adopted for the two wouldbe Higgs doublets. The cross coupling between $H_{1}$ and $H_{2}, \frac{1}{2} N \lambda$, can be calculated from the ladder chain of root vectors. For $G_{2}$ and $F_{4}$, there are two possible root triangles that can be adopted for 2HDM. For each realization, $\vec{y}$ has to be normalized accordingly, thus two possible $N$ 's for $G_{2}$ and $F_{4}$. Although the origins are totally different, Eq.(77) is accidentally identical to the $D$-term Higgs potential in the Minimal Supersymmetric SM(MSSM) if one substitutes $\frac{1}{2} \lambda \Longrightarrow \frac{g^{2}+g^{\prime 2}}{8}$, and $\frac{1}{2} N \lambda \Longrightarrow \frac{g^{2}}{4}$. It is thus expected that the $2 \mathrm{HDM}$ in GHU and the MSSM share a similar physical Higgs mass spectrum.

Among all the Lie groups, the $G_{2}$-based GHU models have the richest $2 \mathrm{HDM}$ phenomenology. In addition to the quartic coupling terms given in Eq.(7), the 2HDM based on $G_{2}$ can have two extra possible forms for $V_{4}$ :

$$
\begin{aligned}
& V_{4}\left(H_{1}, H_{2}\right)=\frac{1}{2} \lambda\left[\frac{1}{3}\left(H_{1}^{\dagger} H_{1}\right)^{2}+\left(H_{2}^{\dagger} H_{2}\right)^{2}\right. \\
& \left.+N_{1}\left(H_{1}^{\dagger} H_{1}\right)\left(H_{2}^{\dagger} H_{2}\right)+N_{2}\left(H_{1}^{\dagger} \sigma^{a} H_{2}\right)\left(H_{2}^{\dagger} \sigma^{a} H_{1}\right)\right]
\end{aligned}
$$

where $\left(N_{1}, N_{2}\right)$ is either $(-2,+1)$ ( as discussed in [10]) or $(+4,-2)$, corresponding to the yellow and red triangles in Fig. 10n the opposite sides or on the same side respectively. For $G_{2}, \vec{y}$ aligns with the medians of two possible root triangles. Hence only one of the two Higgs doublets can acquire the desired hypercharge. In Eq.(8), $H_{1}\left(H_{2}\right)$ carries hypercharge $1 / 2(3 / 2)$. Therefore, only $H_{1}$ is responsible for the EWSB, and $\mathrm{H}_{2}$ is not the canonical Higgs doublet with $|Y|=1 / 2$. We note in passing that since the two possible root triangles of $F_{4}$ lie on different planes, not so interesting $V_{4}$ with $\left(N_{1}, N_{2}\right)=(0,0)$ can emerge.

$\sqrt{v_{1}^{2}+v_{2}^{2}} \sim 246 \mathrm{GeV}$, and $\tan \beta=v_{2} / v_{1}$. The masses of CP odd pseudo-scalar $A_{0}$ and charged Higgs $H^{+}$are given by $M_{A_{0}}^{2}=m_{12}^{2} / \sin \beta \cos \beta$ and $M_{H^{+}}^{2}=M_{A_{0}}^{2}-$ $\frac{1}{4} N \lambda v^{2}$, respectively. Similar to MSSM, the tree-level mass of the lightest neutral Higgs has an upper bound, $M_{h} \leq \sqrt{\lambda v^{2}}\left(\sqrt{7 \lambda v^{2}} / 2\right)$ for $\mathrm{N}=1$ and $4(\mathrm{~N}=7)$, which may causes a phenomenological problem. However, just like in MSSM, there are many heavy degrees of freedom beyond SM in the GHU model. Similar to the positive $\delta M_{h}$ due to the stop loops in the MSSM [11], it is well known that the inclusion of all the radiative corrections from either the bulk fields and their KK excitations, the brane-localized fields, or the brane kinematic 
TABLE II: The candidate simple Lie groups, based on which the 6 dimensional GHU model is phenomenologically viable [6], their root vectors $\vec{\alpha}$ and $\vec{y}$ for $S U(2)_{L}$ and $U(1)_{Y}$ respectively, and all other relevant numbers, see text. Here, $\alpha^{k}$ is the $k-$ th simple root labelled by the Dynkin diagram, and $\tilde{\mu}_{k}$ is the rescaled $k$-th fundamental weights such that $\vec{\alpha}^{i} \cdot \tilde{\mu}_{j}=\delta_{i j}$. Note that the SM quark representation cannot be accommodated in the GHU models based on $C_{n}$ or $D_{n}$ group [6]. However $C_{n}$ and $D_{n}$ are listed here for the sake of comparison and completeness.

\begin{tabular}{|c||c|c|c||c|c|c||c|}
\hline \hline Group & $\alpha$ & $\mathrm{y}$ & $\tan \theta_{W}$ & $\tilde{N}_{-\gamma, \beta}^{2}$ & $\tilde{N}_{\gamma, \beta}^{2}$ & $\theta_{\beta, \gamma}$ & $N$ \\
\hline \hline$A: S U(3 l)$ & $\alpha^{1}$ & $\tilde{\mu}_{2} / 2$ & $\sqrt{3 l /(3 l-2)}$ & $1 / 2$ & 0 & $60^{\circ}$ & 1 \\
\hline$B: S O(2 n+1)$ & $\alpha^{1}$ & $\tilde{\mu}_{2} / 6$ & $\sqrt{3}$ & 1 & 1 & $90^{\circ}$ & 4 \\
\hline$C: U S p(2 n)$ & $\alpha^{n}$ & $\tilde{\mu}_{n-1} / 2$ & $\sqrt{1 /(n-1)}$ & 1 & 1 & $90^{\circ}$ & 4 \\
\hline$D: S O(2 n)$ & $\alpha^{1}, \alpha^{n, n-1}$ & $\tilde{\mu}_{2} / 2, \tilde{\mu}_{n-2} / 2$ & $\sqrt{2 / n-1)}$ & $1 / 2$ & 0 & $60^{\circ}$ & 1 \\
\hline$G_{2}$ & $\alpha^{1}$ & $\tilde{\mu}_{2} / 2, \tilde{\mu}_{2} / 6$ & $\sqrt{1 / 3}, \sqrt{3}$ & $1 / 2,3 / 2$ & 0,2 & $60^{\circ}, 120^{\circ}$ & 1,7 \\
\hline$F_{4}$ & $\alpha^{1}$ & $\tilde{\mu}_{2} / 2, \tilde{\mu}_{2} / 6$ & $\sqrt{1 / 3}, \sqrt{3}$ & $1 / 2,1$ & 0,1 & $60^{\circ}, 90^{\circ}$ & 1,4 \\
\hline$E_{6}$ & $\alpha^{1,5}$ & $\tilde{\mu}_{2,3} / 2$ & $\sqrt{3 / 5}$ & $1 / 2$ & 0 & $60^{\circ}$ & 1 \\
\hline$E_{7}$ & $\alpha^{1,7}$ & $\tilde{\mu}_{2,3} / 6$ & $\sqrt{3}, \sqrt{3 / 2}$ & $1 / 2$ & 0 & $60^{\circ}$ & 1 \\
\hline$E_{8}$ & $\alpha^{1,8}$ & $\tilde{\mu}_{2,3} / 6$ & $\sqrt{9 / 7}, \sqrt{3 / 5}$ & $1 / 2$ & 0 & $60^{\circ}$ & 1 \\
\hline
\end{tabular}

terms could largely enhance $M_{h}$ from the above tree-level prediction [12]. However, a general discussion on this issue is still lacking. Such a model-independent discussion on the radiative corrections to $M_{h}$ is beyond the scope of the present paper, here we just assume that a careful consideration which includes the radiative corrections to $V_{4}$ can rescue the lightest neutral Higgs mass problem as in MSSM. The investigation along this line will be presented elsewhere 13 .

Finally, we address the weak mixing angle problem in GHU which is independent of $d$. Adding the BKT to GHU model is one of the remedies to obtain a realistic $\sin ^{2} \theta_{W}$ close to experimental value. We use the following BKT which involves gauge zero modes on the brane only,

$\mathcal{L}_{B . K}=-\frac{1}{4} \int d^{4} x d x^{5} d x^{6} \delta\left(x^{5}\right) \delta\left(x^{6}\right)\left[c_{1}\left(F_{\mu \nu}^{(0) a}\right)^{2}+c_{2}\left(F_{\mu \nu}^{(0) b}\right)^{2}\right]$

where the superscript (0) represents zero mode, $c_{1}, c_{2}$ are free parameters with mass dimension -2 , and $a, b$ are the group indices for SM $S U(2)_{L}$ and $U(1)_{Y}$, respectively. The $4 \mathrm{D}$ effective gauge couplings are modified:

$$
g_{4 D}^{i} \rightarrow \frac{g_{4 D}^{i}}{\sqrt{Z_{i}}}, \quad \text { with } \mathrm{Z}_{i}=1+\frac{c_{i}}{\mathrm{Z}_{0}^{2}}, \quad(\mathrm{i}=1 \text { or } 2),
$$

where $Z_{0}^{2}$ is the volume of the two extra dimensions. As a result, the weak mixing angle becomes

$$
\tan \theta_{W} \rightarrow \sqrt{\frac{Z_{1}}{Z_{2}}} \tan \theta_{W} .
$$

From the experimental value $\tan \theta_{W}=0.5356$ [14], the ratio of $Z_{1} / Z_{2}$ can be fixed for a given $G_{M}$. Moreover, the upper bound of the lightest neutral Higgs mass gets a factor of $\sqrt{Z_{1}}$ enhencement and this problem can be alleviated as well.

Adding an additional $U(1)^{\prime}$ gauge group is an alternative to obtain a realistic weak mixing angle in GHU model. The mixing between SM $U(1)_{Y}$ and $U(1)^{\prime}$ leads to an effective $Z_{2} \neq 1$ ( with $Z_{1}=1$ ) as in the previous case. The extra $U(1)$ factor introduces a new electrically neutral gauge boson whose mass shall be arranged to be the order of the compactification scale. The original $\tan \theta_{W}$ for each group without $U(1)^{\prime}$ can be found in table [II Note that to obtain the realistic $\theta_{W}$, $S U(3), S O(2 n+1), G_{2}, F_{4}$ and $E_{7}$ require the largest $Z_{2}$ among all the groups.

Before concluding, we remark on the new scalar boson recently observed near $126 \mathrm{GeV}$ with the diphoton excess at the large hadron collider (LHC) [15, 16]. Although most of its physical properties seem to be consistent with the elementary Higgs boson in the SM, the diphoton excess may indicate the existence of new physics beyond the SM. As discussed, $M_{h} \sim 126 \mathrm{GeV}$ could be easily accommodated in a realistic $2 \mathrm{HDM}$ in the GHU models with some construction dependent extensions. We note in passing that the diphoton excess, if persist, could also be explained in this GHU framework for there are many charged heavy degrees of freedom.

In summary, we perform a general group theory analysis on the realization of $2 \mathrm{HDM}$ in GHU model with a 6 -dimensional gauge $G_{M}$ symmetry, where $G_{M}$ is a simple Lie group. We showed that a $2 \mathrm{HDM}$ at low energy can possibly be made if the three root vectors associated with the would-be Higgs doublets and the SM $S U(2)_{L}$ form isosceles triangles with vertex angle either of $\pi / 3, \pi / 2$ or $2 \pi / 3$. The quartic coupling terms in the $4 \mathrm{D}$ effective $2 \mathrm{HDM}$ potential are completely determined by the group $G_{M}$ at tree-level. Only a few potential forms can be admitted for all possible Lie groups, as shown in Table III Moreover, which form to be admitted depends solely on the vertex angle. We observed that, among all possible Lie groups, the 2HDM based on the GHU model with $G_{2}$ gauge symmetry has the richest structure in the Higgs potential. We discuss the mass spectrum of physical Higgs bosons and two possible 
remedies to obtain a realistic weak mixing angle in GHU models as well. Finally, we briefly comment how to accommodate the recent observed $\sim 126 \mathrm{GeV}$ scalar boson at the LHC in this GHU framework.

W.F.C. was supported by the Taiwan NSC, Grant No. 99-2112-M-007-006-MY3. S.K.K was supported by NRF and MEST of Korea, No. 2011-0029758. J. P. was supported by the Taiwan NSC, Grant No. 100-2811-M-007030 and 099-2811-M-007-077. J.P. thanks D.W. Jeong for valuable comments and discussion.

* Electronic address: wfchang@phys.nthu.edu.tw

† Electronic address: skkang@snut.ac.kr

$\ddagger$ Electronic address: honolo@phys.nthu.edu.tw

[1] N. S. Manton, Nucl. Phys. B 158, 141 (1979). D. B. Fairlie, Phys. Lett. B 82, 97 (1979). P. Forgacs and N. S. Manton, Commun. Math. Phys. 72, 15 (1980).

[2] Y. Hosotani, Annals Phys. 190, 233 (1989). Y. Hosotani, Phys. Lett. B 126, 309 (1983); ibid. 129 (1983) 193.

[3] I. Antoniadis and K. Benakli, Phys. Lett. B 326, 69 (1994).

[4] C. A. Scrucca, M. Sernoe, A. Wulzer and L. Silvestrini, JHEP 0402, 049 (2004).

[5] B. Grzadkowski and J. Wudka, Phys. Rev. Lett. 97, 211602 (2006) arXiv:hep-ph/0604225.

[6] A. Aranda and J. Wudka, Phys. Rev. D 82, 096005
(2010) arXiv:1008.3945 [hep-ph]].

[7] G. Burdman and Y. Nomura, Nucl. Phys. B 656, 3 (2003) arXiv:hep-ph/0210257. K. Agashe, R. Contino and A. Pomarol, Nucl. Phys. B 719, 165 (2005) arXiv:hep-ph/0412089. A. Aranda and J. L. Diaz-Cruz, Phys. Lett. B 633, 591 (2006) arXiv:hep-ph/0510138. G. Panico, M. Safari and M. Serone, JHEP 1102, 103 (2011) arXiv:1012.2875 [hep-ph]].

[8] J. Park and S. K. Kang, JHEP 1204, 101 (2012) arXiv:1111.5422 [hep-ph]].

[9] R. Gilmore, "Lie groups, Lie algebras, and some of their applications", Dover edition (2005)

[10] C. Csaki, C. Grojean and H. Murayama, Phys. Rev. D 67, 085012 (2003) hep-ph/0210133.

[11] H. E. Haber and R. Hempfling, Phys. Rev. Lett. 66, 1815 (1991); Y. Okada, M. Yamaguchi and T. Yanagida, Prog. Theor. Phys. 85, 1 (1991); Phys. Lett. B262, 54 (1991). J. Ellis, G. Ridolfi and F. Zwirner, Phys. Lett. B257, 83 (1991); Phys. Lett. B262, 477 (1991).

[12] I. Antoniadis, K. Benakli and M. Quiros, New J. Phys. 3, 20 (2001) arXiv:hep-th/0108005. C. A. Scrucca, M. Serone and L. Silvestrini, Nucl. Phys. B 669, 128 (2003) arXiv:hep-ph/0304220. G. Cacciapaglia, C. Csaki and S. C. Park, JHEP 0603, 099 (2006) arXiv:hep-ph/0510366.

[13] work in progress.

[14] K. Nakamura et al. [Particle Data Group], J. Phys. G 37, 075021 (2010).

[15] G. Aad et al. [ATLAS Collaboration], Phys. Lett. B 716, 1 (2012) arXiv:1207.7214 [hep-ex]].

[16] S. Chatrchyan et al. [CMS Collaboration], Phys. Lett. B 716, 30 (2012) arXiv:1207.7235 [hep-ex]]. 